\title{
Permanence of the information given during oncogenetic counseling to persons at familial risk of breast/ovarian and/or colon cancer
}

\begin{abstract}
Fabrice Kwiatkowski ${ }^{\star, 1}$, Pascal Dessenne ${ }^{1}$, Claire Laquet ${ }^{1}$, Marie-Françoise Petit ${ }^{1}$ and Yves-Jean Bignon ${ }^{1}$
How long counselees retain the information given during their genetic consultation is of major importance. To address this issue, we conducted a survey among the $\mathbf{3 5 0 0}$ families that have been offered genetic counseling at our Center since $\mathbf{1 9 8 8}$. In August 2007, we mailed a questionnaire to a representative subset of 579 persons belonging to breast/ovarian or colon cancer families seen in the last 10 years, either carrying an identified mutation or not. Targeted topics included the meaning of hereditary predisposition, the medical prevention related to the familial risk, the steps to undertake for a new family member to enter the genetic testing program and general knowledge of hereditary predisposition to cancer. A total of 91 randomized non-respondents were sent a second, more inciting letter, in order to assess any non-response bias. Overall, 337 questionnaires were collected: response rate was $58 \%$. Standardized average knowledge was $7.28 \pm 1.52$ of 10 . Scores were lowest concerning medical prevention. The level of knowledge decreased with age $\left(P<10^{-6}\right)$, but increased with educational level $\left(P<10^{-5}\right)$ and mutation status $(P=0.01)$. Surprisingly, no erosion of patients' knowledge over the time was observed $(P=0.41)$. Among persons at hereditary risk of colon cancer, the level of knowledge tended to improve with time, in contrast to the breast/ovarian group $(\boldsymbol{P}=\mathbf{0 . 0 1 7 )}$. Among persons with a familial risk of breast/ovarian or colon cancer, a renewal of oncogenetic counseling does not seem necessary to maintain the level of specific knowledge. Measures to help patients follow their medical prevention, as organizing or checking their medical examinations, seem indicated.
\end{abstract}

European Journal of Human Genetics (2012) 20, 141-147; doi:10.1038/ejhg.2011.169; published online 21 September 2011

Keywords: oncogenetics; information; cancer; genetic counseling; survey

\section{INTRODUCTION}

Oncogenetic counseling for predictive genetic testing has existed for more than 20 years in France, and the comprehensive cancer hospital Jean Perrin in Clermont-Ferrand has been a pioneer in that domain, beginning this activity in 1988. Since then, more than 3500 families have been accrued, mainly at risk of breast, ovarian and colon cancer, and about 100000 persons belonging to these families have been registered in our database. Measures of the psychological impact of predictive genetic counseling have been of interest for many years. ${ }^{1}$ Other more recent reviews confirm the relatively mild emotional consequences of predictive genetic testing and better knowledge of the recipient. ${ }^{2,3}$ The follow-up period of these studies was very short, however, often less than 2 years, which limits the range of conclusions to be drawn. The importance of the consultation content has been explored. ${ }^{4}$ It has been shown that the satisfaction of the recipients was mainly dependent on the quality and quantity of information given by the consultant, and that the evaluation of coping strategies of recipients was essential to minimize emotional consequences. As recipients are expected to transmit their informations to relatives, these emotional consequences are not limited to the counseling itself but they may last long after. To report a mutation discovery has very often negative impacts on familial relationships and on the whole communication within the familial circle. ${ }^{5-7}$ Moreover, information of other members of the family may be impaired by insufficient knowledge of transmitters ${ }^{8}$ and/or a wrong understanding of the hereditary cancer risk. ${ }^{9}$

The evolution of the recipients' knowledge is thus very important. It may influence two major points:

- The recipient's compliance with the recommendations given, and therefore the quality of his/her medical surveillance.

- The extension of medical management to relatives (recruitment of new family members), insofar as he can communicate correct information and persuade them to request genetic testing and counseling.

The persistence of recipients' genetic knowledge has been addressed in very few studies: most of them concerned the short-term recall of the delivered information (1-6 months after consulting). ${ }^{10-12}$ In two others, ${ }^{13,14}$ the delay was longer (1-5 years after consultation) and targeted women at breast/ovarian cancer risk, but studied information was limited to individual and offspring cancer risk, heredity likelihood and/or early detection schemes: the persistance of information was found to be poor and depended on the mutational status of recipients. The survey of Somer et $a l^{15}$ was the oldest one (1988): it tested a large sample of counselees $(N=791)$ but the matter of interest was the consequences of the diagnosis on attitudes toward reproduction, prenatal study and abortion, and did not focus on genetic mutations 
related to long-term cancer predispositions. Concerning the retention of information, these authors concluded that most of the essential facts were correctly recalled and no significant decrease in time could be noticed.

As adequate oncogenetic knowledge is mainly obtained during one or two consultations with specialists, we suspected as some of these authors that it would likely fade away in time, and thus questioned the necessity to renew the recipients' genetic information through periodic consultations. The long follow-up of numerous consulting families in our hospital, as their members had not consulted for years, enabled us to evaluate the permanence of their knowledge. To obtain information about whether and how recipients' knowledge was fading, and at what frequency a renewal of their information might be useful, we conducted a survey among a representative sample of almost 600 individuals belonging to families at risk of breast, ovarian and/or colon cancer.

\section{SUBJECTS AND METHODS}

Genetic counseling at our center involves a geneticist, a physician, a genetic counselor (nurse), a psychologist and a secretarial service. An engineer is responsible for the computer management of medical data. After an initial contact by telephone patients are asked to describe their genealogical tree, indicating cancers and other possible hereditary diseases. In an initial consultation, patients present themselves and their pedigree to the genetic counselor, who helps the patients complete their genealogical tree and informs them about hereditary cancer risk and the genetic testing process. The patients then meet the geneticist, who evaluates the hereditary risk. If genetic testing is indicated, the patients are provided with further medical information and a first-blood sample is taken (a second one is required later if a mutation is found). An information booklet concerning the type of familial risk is offered, and a letter is sent to their general practitioner (or other physician if they wish) to inform them about the onset of genetic testing.

Once the analysis is completed, the patient returns for a second pair of consultation, with the genetic counselor and then with the geneticist or the physician. The genetic counselor updates the pedigree, if needed, with the patients and evaluates their global knowledge and their capacity to cope with the announcement of the result. Two types of consultation are possible with the geneticist/physician:

- An individual mode where the result is given to the person alone.

- A familial mode where the results of the genetic analysis (positive or negative) are given to several members of a family, but without telling who is a carrier. This type of consultation is indicated when the whole result is negative or when the carrier of the found mutation is not present. Actually, this latter mode is less and less used as it has shown some limits.

Personal cancer risk is explained and recommendations are given concerning suitable medical supervision and the necessity to encourage relatives to ask for genetic testing in order to be benefited from effective screening and prevention. A final letter is then addressed to the patient(s) and his/her designated doctor(s). All along the research, the recipients are informed that they can always ask for a consultation with the psychologist.

\section{Database of familial information}

An updated version of SEM software ${ }^{16}$ was used to extract a representative sample of probands and relatives from our genetic consultation database. This version stores standard information, including gender, birth date, address, marital status and also medical data such as date of past consultations, type of mutation (if any), cancer (if any) location and age at diagnosis, disease status and date of death.

\section{Development of the questionnaire}

A committee of experts (geneticists, physicians, psychologists, nurses and statistician) was designated to elaborate a questionnaire that could answer to defined questions. The face and content validity was assessed by a group of oncologists, radiotherapists, surgeons, nurses, health supervisors, secretaries, biologists and patients. Questions were either direct (addressed to the respondent) or indirect (general purpose, use of the third person) and sometimes partially redundant to facilitate cross-controls.

Questions were grouped into several sections:

- Marital status, children, activity, educational level and cancer history

- Date of last genetic counseling session

- Present compliance with medical recommendations

- General knowledge of the meaning of hereditary predisposition to cancer

- Necessary steps for a new family member to obtain genetic testing

- General knowledge about screening, genetic mutations and their consequences

- How transmission of genetic information is done or inhibited within the family

- Difficulties in putting recommendations into practice

- Remarks on the breast/ovarian cancer information booklet

In all, 10 questions concerned the section 'current compliance with medical recommendations', and presented a series of possible examinations (mammography, MRI, clinic, ultrasound, trans-vaginal ultrasound, cervical smear, aspirative smear, colonoscopy, occult bleeding, blood markers dosage, etc) for which the subject was asked to specify the recommended frequency in relationship with his/her cancer risk. Available answers ranged from 6 months to never, including 6, 12, 18 and 24 months. Correctness scoring was adjusted according to the history of cancer of each subject. Answers were counted as correct if they applied to the subject.

The fourth section tested knowledge of the meaning of hereditary predisposition. Seven questions proposed degrees of agreement with various propositions, including

- a supplementary risk of cancer for yourself, your family;

- predisposition to a certain kind of cancer for yourself, your family;

- a diagnosis of cancer;

- a vulnerability to environmental factors;

- a risk of infection.

The last three topics were considered false, although some could argue whether mutations associated with cancer represent a possible weakness to environmental threats.

The fifth section, of 11 questions, grouped propositions concerning the necessary steps for someone to start a personal genetic inquiry. One or two consultations with a geneticist were the first questions. Different types of biological samples were then proposed: saliva, urine, skin, blood (one or two samples), hair, semen (for men) and lumbar puncture. A scan or RMI was the last item. According to our actual genetic testing process, good answers were two consultations and two blood samples, as a mutation discovery must be certified by identical results on two different blood samples obtained at two different times. But one consultation and a single blood sample were also rated correct since years ago, this was the recommendation.

The sixth chapter entitled 'General knowledge about screening, genetic mutations and their consequences', comprised 24 proposals, all presented with a Likert scale enabling to quote the degree of agreement. They were labeled as possibly true or false sentences, as for example:

- Some cancers are hereditary.

- If one has a familial predisposition, it means he would necessarily have a cancer.

- A genetic mutation transmits itself more often between a parent and children of the same sex.

- The cancer is not hereditary, but the predisposition is.

- Obtaining my personal genetic test can increase my cancer risk.

Further sections are not treated in this report.

\section{Inclusion and response diagram}

After approval by the local ethics committee, the anonymous questionnaire was mailed to a representative subset of 579 probands and relatives from our 


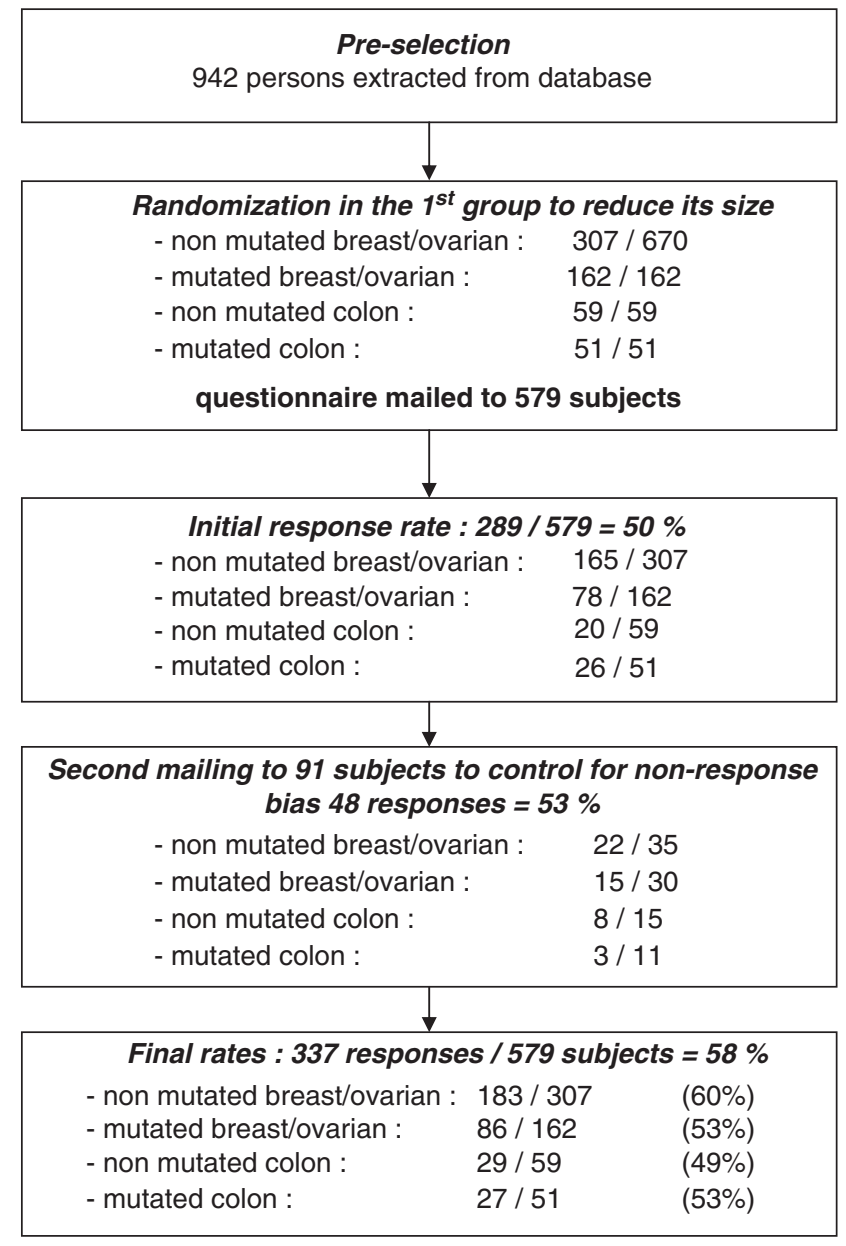

Figure 1 Flowchart of survey accrual. Numbers indicate responses/ questionnaires sent.

database, with a letter to introduce the survey signed by the geneticist. In order to limit heterogeneity, only breast/ovarian and colon cancer risks were targeted.

The pre-selection search of the database identified for living persons registered in families at risk of breast, ovarian and/or colon cancer, with a genetic counseling consultation in the last 10 years, older than 18 years and with a known address. A randomization was performed within the nonmutated breast/ovarian group in order to reduce its size, but in other groups we kept all the subjects in order not to reduce analysis power. So, no exclusions were made in mutation-carrier groups, as these categories were smaller (Figure 1). To assess possible non-response bias, a second mailing was sent to one third of a randomized subset of non-respondents.

\section{Survey design and statistics}

The number of persons contacted was calculated to provide $\pm 5 \%$ precision in the results (range of the $95 \%$ confidence interval), which required 400 responses. A final response rate of 58\% was achieved with 337 answered mails over 579 , thus 63 fewer than the 400 expected. The accuracy of the main results was therefore $\pm 5.3 \%$, which remained acceptable.

Answers to the survey questions are treated in a quantitative or qualitative manner as appropriate. Most were scored on four-level Likert scales (from total disagreement to complete agreement) in order to force respondents to choose one side or the other (with a five-level scale, many persons select the median class). Likert scale results were transformed into scores and confidence intervals calculated, using 0 for total disagreement, 1 for mild disagreement, 2 for mild agreement and 3 for maximal agreement.
When answers reflected a state of knowledge from other sources, or when they were compared with information given during oncogenetic counseling, they generally were either right or wrong, making it possible to group questions of a section and compute an average 'grade' over 10 that measured the correctness of the subjects' answers. The larger the gap was between this grade and the optimum of 10 , the less knowledge the subject retained for the topic. Sections containing medical or practical information were treated in this way.

For statistical testing, standard repartition parameters (numbers, means, medians, standard deviations and ranges) were calculated. Tests used to study the link between pairs of variables consisted of $\chi^{2}$, one-way ANOVA or Kruskal-Wallis $\mathrm{H}$ test (depending on normality of distributions and/or difference of variances) and Pearson's correlation coefficient or Spearman's rank tests. Intra-subject comparisons were performed using paired tests.

Standard $P$-values less than 0.05 were used as the threshold for significance. Statistical tests were performed using the SEM software. ${ }^{16}$

\section{RESULTS}

Population characteristics

Surveyed subjects all resided in the region surrounding the Center where genetic counseling was performed, with 54\% living in the department and $46 \%$ in neighboring departments. The response rate was not related to distance from the Center $(P=0.83)$.

In all, $86 \%$ of the respondents were female (Table 1 ) as expected, as the main indication for genetic counseling was breast/ovarian cancer risk and analysis of the BRCA1 and BRCA2 genes.

A total of $56 \%$ of respondents had a personal history of cancer, as this was the main reason for seeking genetic consultation: $83 \%$ were breast cancers, $8 \%$ colon, $4 \%$ ovarian, and endometrial, thyroid, skin, prostate, stomach, bladder, lymphoma and lung at less than $2 \%$. Half (166/337) occurred in families with a mutation, though 44 of these did not carry the familial mutation.

Median delay after genetic counseling was 1.5 years. The yearly distribution of this delay is shown in Figure 2.

For $40 \%$ of subjects, genetic counseling occurred the year before the survey. Only 53 questionnaires $(16 \%)$ were obtained with more than 3 years follow-up. Because of randomization, this distribution reflected our global population accrual. But it could have been relevant to try to balance the older patients' classes with a different sampling method.

\section{Bias analysis}

Although the response rate did not significantly differ between distant and recent consultations $(P=0.12)$, the answer rate was $11 \%$ lower when the delay after counseling was over 3 years (52 versus $41 \%$ ). Also, older persons, males, persons with no history of cancer and/or at risk of cancers other than breast/ovary did not answer as frequently, but none of these tendencies were significant (Table 2).

Comparison of the response rate with educational level was not available, as we did not have this information for non-respondents.

Comparison between respondents to the first and second mailings gave slightly different results. Respondents to the second mailing were a bit older ( +5.3 years, $P=0.044)$, but their educational level and employment status were similar ( $P=0.70$ and 0.86 , respectively). A profession in the medical domain did not change the response rate $(P=0.35)$. One difference appeared concerning the question 'did the genetic counseling change your perception of your cancer risk': among first responders, $53 \%$ said yes, versus $72 \%$ of second responders $(P=0.027)$. Other criteria added nothing to previous comparisons between respondents and non-respondents.

\section{Measures of subjects' knowledge}

Figure 3 shows the scores obtained from all subjects. 
Table 1 General characteristics of subjects (for categorical parameters numbers and percentages are given, average \pm standard deviation (range) for quantitative ones)

\begin{tabular}{|c|c|}
\hline Items & Distribution parameters \\
\hline \multicolumn{2}{|l|}{ Gender } \\
\hline Female & $291(86.4 \%)$ \\
\hline Male & $46(13.6 \%)$ \\
\hline \multicolumn{2}{|l|}{ Age (at survey) } \\
\hline Mean & $52.5 \pm 14.6$ (SD) \\
\hline Range & $(18-82)$ \\
\hline \multicolumn{2}{|l|}{ Marital situation } \\
\hline Lives in couple & 249 (77.6\%) \\
\hline Other & $72(22.4 \%)$ \\
\hline Number of children & $1.7 \pm 1.1(0-5)$ \\
\hline \multicolumn{2}{|l|}{ Educational level } \\
\hline Primary & $61(19.2 \%)$ \\
\hline High school & $137(43.3 \%)$ \\
\hline University & $119(37.5 \%)$ \\
\hline \multicolumn{2}{|l|}{ Employment } \\
\hline Employed & $168(50.1 \%)$ \\
\hline Retired & $115(34.3 \%)$ \\
\hline Homemaker & $14(4.2 \%)$ \\
\hline Unemployed & $3(0.9 \%)$ \\
\hline Other (disabled...) & $37(10.5 \%)$ \\
\hline \multicolumn{2}{|l|}{ Profession related to medicine } \\
\hline No & $264(84.6 \%)$ \\
\hline Yes & $48(15.4 \%)$ \\
\hline Time from genetic counseling & 1.8 years $\pm 1.7(0-9)$ \\
\hline \multicolumn{2}{|l|}{ Personal mutation status } \\
\hline Mutated & $122(36.2 \%)$ \\
\hline Not mutated & $215(63.8 \%)$ \\
\hline \multicolumn{2}{|l|}{ Belongs to a mutated family } \\
\hline Yes & $166(49.3 \%)$ \\
\hline No & $171(50.7 \%)$ \\
\hline \multicolumn{2}{|l|}{ Familial cancer risk } \\
\hline Breast & $280(83.1 \%)$ \\
\hline Colon & $57(16.9 \%)$ \\
\hline \multicolumn{2}{|l|}{ Pathological history } \\
\hline None known & $136(40.3 \%)$ \\
\hline Benign & $12(3.6 \%)$ \\
\hline Cancer & $189(56.1 \%)$ \\
\hline
\end{tabular}

General information scored highest, with a mean of 7.92. Knowledge of medical intervention scored lowest, at 6.85 . The mean value of the four scores was $7.28 \pm 1.52$ (standard deviation).

This global level of knowledge was higher when among mutation carriers $(P=0.021)$, and those belonging to a mutated family $(P=0.014)$, among subjects still working $(P=0.00015)$ and if their profession was related to the medical domain $(P=0.05)$, and those

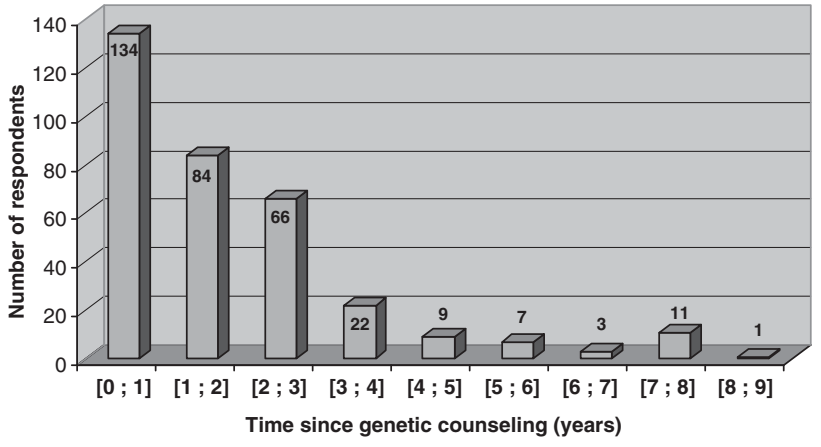

Figure 2 Delay between genetic counseling and the survey.

Table 2 Characteristics of responders and non-responders

\begin{tabular}{lcccc}
\hline & No answer & To 1st mailing & To 2nd mailing & P-value \\
\hline Number of subjects & 242 & 289 & 48 & \\
Age at survey & $54.4 \pm 14.7$ & $52.9 \pm 14.3$ & $58.2 \pm 12.0$ & 0.11 \\
Males & $17.7 \%$ & $13.1 \%$ & $16.7 \%$ & 0.22 \\
Cancer history & $47.3 \%$ & $56.0 \%$ & $56.1 \%$ & 0.11 \\
Mutated & $37.3 \%$ & $36.0 \%$ & $37.5 \%$ & 0.94 \\
Breast/ovarian cancer risk & $78.0 \%$ & $84.1 \%$ & $77.1 \%$ & 0.16 \\
Number of children & $1.71 \pm 1.28$ & $1.52 \pm 1.13$ & $1.54 \pm 1.22$ & 0.33 \\
Married & - & $77.3 \%$ & $79.0 \%$ & 0.80 \\
Average delay after & $2.26 \pm 2.31$ & $1.78 \pm 1.68$ & $2.16 \pm 1.77$ & 0.12 \\
consulting (years) & & & & \\
\hline
\end{tabular}

aThis probability corresponds to tests comparing all three means or proportions in the same time.

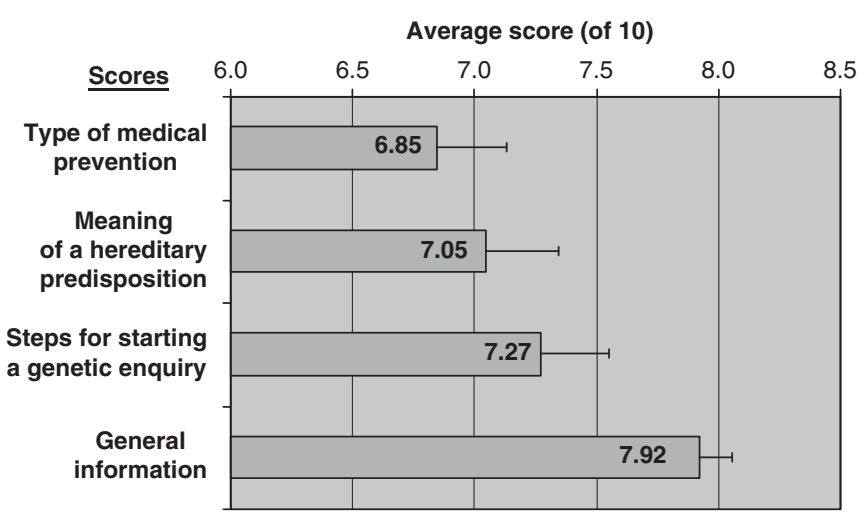

Figure 3 Mean scores with 95\% confidence intervals for main topics.

with higher educational level $(P=0.000004)$. The global score was negatively correlated to age (0.000006), although obviously, younger respondents were also those still working.

No relationship was found between this mean score and the type of cancer risk (breast/ovarian or colon), answering the second mailing, gender, marital status, number of children or the discovery of new cancers in the family since the last genetic consultation.

\section{Evolution of subjects' knowledge}

The mean knowledge score did not change with increasing delay between the date of the genetic counseling and the survey 


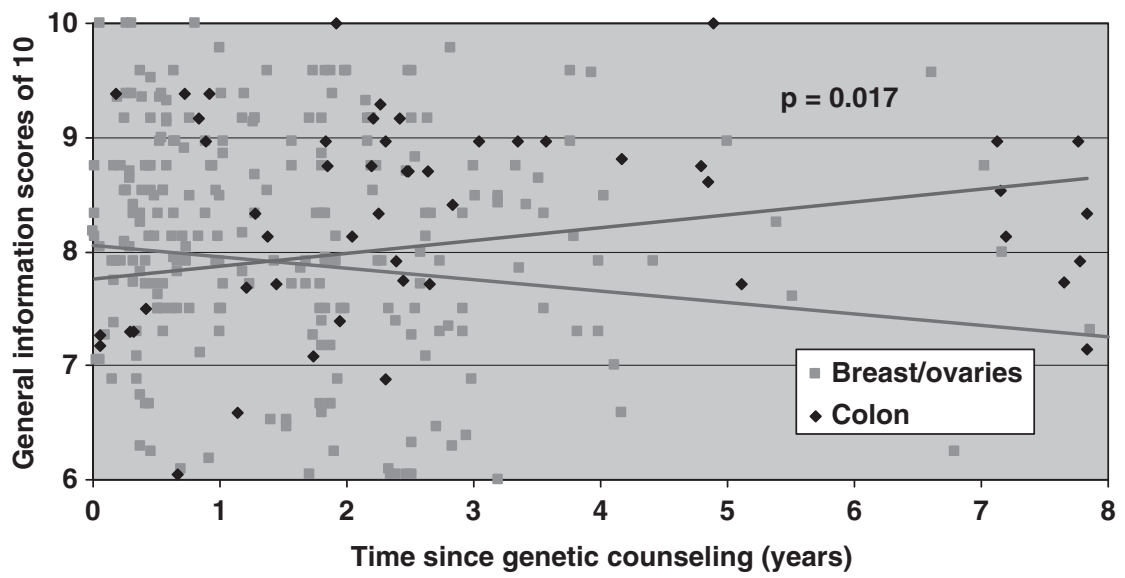

Figure 4 Evolution differences of knowledge levels along time since genetic counseling according to cancer risk location.

$(r=-0.009$, not significant (NS)). The lack of correlation was confirmed for each sub-scale: the type of medical prevention (correlation coefficient $r=-0.053$, NS), the meaning of hereditary predisposition $(r=0.056, \mathrm{NS})$, the steps to start a personal genetic inquiry $(r=-0.045, \mathrm{NS})$ and the general information score $(r=-0.011$, NS). This stability did not vary among classes of age (cut-off $=60$ years), although the regression line for older people was significantly lower than for younger people $(P=0.0075)$. Mutation carrier status did not either impact this stable trend $(P=0.48)$.

The only parameter tending to influence the evolution of knowledge was the type of cancer risk ( $P=0.06$ for the global score), especially (Figure 4$)$ for the general information subscale $(P=0.017)$. Subjects with breast/ovarian hereditary risk showed a rather decreasing regression line through time $(r=-0.12, P=0.08)$, while colon cancer risk was associated to an increasing line $(r=0.22, P=0.05)$. But this difference was not due to sex, although sex was related to cancer risk location.

\section{Differences between specific and non-specific knowledge}

Following the reviewers' suggestions, two types of knowledge were separately scored:

- A specific one characterizing genetic data related to the hereditary predisposition. It comprised 15 items of the 'general information' subscale.

- A non-specific knowledge gathering the other nine questions that had a more general scope.

Although these two types of information significantly differed in average $(8.1 \pm 1.2$ for the specific information versus $8.7 \pm 1.2$ for the more general one; $P<10^{-7}$ ), no trend in time could be noticed and thus no difference between the two slopes which were close to zero $(P=0.51)$. Both knowledge types were associated to educational level ( $P=0.000002$ and $P=0.0002$, respectively). Concerning the nonspecific information, mutated subjects did not differ from nonmutated ones $(P=0.11)$ but a slightly higher level of specific knowledge characterized the mutated individuals $(8.3 \pm 1.2$ versus $8.0 \pm 1.1$ for non-mutated ones, $P=0.018$ ). The same analysis depending on the type of cancer risk (breast/ovarian or colon) did not increase discrepancies.

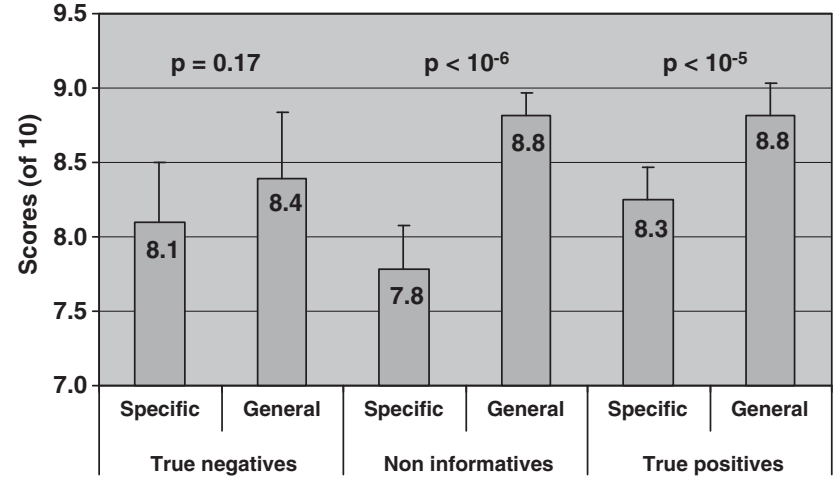

Figure 5 Mean scores with 95\% confidence intervals for specific and nonspecific knowledge within non-mutated individuals (true negatives, noninformatives) and true positive ones (ie mutated).

A special attention in relationship with both types of knowledge was paid to the non-mutated group: this latter gathered two kinds of individuals:

- The true-negatives: they were tested negative although they belong to families where a deleterious mutation exists. They have the same cancer risk as the general population.

- The non-informative individuals: they belong to these at-risk families where no known mutation is discovered. Their cancer risk is high although they cannot be sure they have inherited any mutation.

These two groups of individuals seemed to behave differently (Figure 5): for the true-negative persons, the level of specific knowledge $(8.1 \pm 1.2)$ was quite similar $(P=0.17)$ to the level of their general knowledge $(8.4 \pm 1.3)$. For non-informative persons, both scores were not alike $\left(P<10^{-6}\right)$ : means equaled, respectively, $7.8 \pm 1.1$ and $8.8 \pm 1.1$. Using the ratio specific over non-specific knowledge, true-negatives seemed to have better understood the specific genetic information than non-informative individuals $(P=0.0055)$. The particularity of non-informative subjects was also confirmed by their lower level of specific knowledge when compared with true-positive individuals (ie mutated): $8.3 \pm 1.2$ for these latter $(P=0.005$ and $P=0.021$ for the comparison of ratio), while 
their level of non-specific knowledge did not differ $(8.8 \pm 1.0$ versus $8.8 \pm 1.2, P=0.49$ ).

\section{DISCUSSION}

This prospective survey belongs to the very few that studied the evolution of genetic knowledge of persons with a cancer hereditary risk. The range of topics surveyed appeared adequate to answer our questions. The $58 \%$ response rate was rather good for a survey sent by mail. Because of the randomization, sample should be representative of all our patients. A second mailing to $1 / 3$ of non-respondents showed no response bias.

Surprisingly, the outcome did not confirm our initial hypothesis: the observed level of knowledge of persons who had undergone genetic counseling did not change with time, although it did depend on their age and educational level. It seems that once they had been informed, the information was retained. The mean value of our scores stands at 7.28 of 10 , meaning $73 \%$ of the information is rather well recalled. This tallies with Michie's statistics ${ }^{10}$ (76\%) and Somer's ones $^{15}(65-86 \%)$ but diverges from DiCastro ${ }^{13}(30-62 \%)$ and Bober $^{12}(10-50 \%)$. This discrepancy may arise from the type of knowledge investigated. Although DiCastro and Bober question about very specific points (cancer risk, heriditary likelihood and prevention clues), we tried to evaluate a larger panel of knowledge. It is also possible that qualitative information may be easier to memorize than quantitative ones. On an other hand, Bober's mode of questioning was very harsh: 'Please, describe all of the recommendations you received during your visit.' This kind of open question leaves the subject helpless. In contrast, a set of questions focusing on each point will favor subjects' recall and probably better evaluate the real level of their knowledge, although it does not guarantee that this knowledge will promote a correct prevention attitude on the long run.

Several reasons may explain the stability we noticed. Probands and their relatives have other sources of information (eg media and internet) that refresh their knowledge. Independently, intra-family communication promotes sharing and renewal of this information. Finally, persons at risk undergo frequent medical supervision that gives as many occasions to learn more about their familial predisposition.

The third point is confirmed by the difference in Figure 4 observed between breast/ovarian cancer risk and colon cancer risk. Indeed, the colonoscopy recommended for persons at risk of colon cancer is much more invasive than mammography and clinical examination indicated for breast cancers, or ultrasonography and cervical smear for ovarian cancers. This may change the global attitude of patients that have to cope with such threats, and influence their search for information.

Considering the results in Figure 5, there may be some good reasons to spend more counseling time with non-informative subjects, that is, the individuals belonging to at-risk families where no known mutation is found. These subjects appeared to have the lowest score of specific knowledge, although they performed very well with the more global information. Perhaps the uncertainty concerning their cancer predisposition background may blur their understanding of specific genetic mechanisms that sustain any hereditary risk.

A possible limitation of this study can be found in its design. Subjects' knowledge was not collected just after genetic counseling and then again years after. Therefore no paired test could be done. Instead, we supposed that recent consultants were representative for the level of knowledge immediately after consultation, and older consultations for knowledge retained over time. As no major changes have been made in the way genetic counseling is provided to patients, this strategy appeared pertinent. This might be discussed, as this 10 -year period has presented contextual changes, for example, with the generalization of internet access and content. Conclusions drawn from this study may be less precise than one assessing the same patients over time, with individual variations remaining invisible. But this is not likely to reverse the global tendency observed in the survey. An other weakness of our study comes from the sampling method used: randomization, necessary to guarantee that no selection bias would intervene in our statistics, has prevented us from having similar population sizes in each period of time. Thus the outcomes in older consultants may lack of acuracy.

The overall conclusion of these results is that it does not appear necessary to renew genetic consultations to keep patients knowledgeable about their genetic risk: the present counseling process seems sufficient to give them the necessary information for their medical follow-up, and for the subjects to transmit information to their relatives. The supply of an audiotape of the consultation, a solution tested by Watson et al, ${ }^{11}$ does not seem either to be useful as it did not help increase the acuracy of retained knowledge. Nevertheless, the lowest-scoring subscale in our survey was the one concerning the medical examinations required for screening and their frequency: a same conclusion can be drawn from Bober et al, ${ }^{12}$ as only $33-43 \%$ of screening information were recalled. This suggests that patients need more help to achieve compliance and perhaps the counseling team could have a more active role in this process. Concerning the subgroup of subjects belonging to families where no known mutation is found, a special attention should be paid to the explanations they receive: it seems that they are less able to understand specific genetic information, as their familial cancer risk remains unexplained.

\section{CONFLICT OF INTEREST}

The authors declare no conflict of interest. The survery was exclusively financed by the Comprehensive Cancer Centre Jean Perrin.

\section{ACKNOWLEDGEMENTS}

We thank Nancy Uhrhammer for the english review; Laurence, Mélanie, Sandie and Sandrine for the data management. We are grateful for the very contributing comments of the reviewers.

1 Broadstock M, Michie S, Marteau T: Psychological consequences of predictive genetic testing: a systematic review. Eur J Hum Genet 2000; 8: 731-738.

2 Braithwaite D, Emery J, Walter F, Prevost AT, Sutton S: Psychological impact of genetic counseling for familial cancer: a systematic review and meta-analysis. J Natl Cancer Inst 2004; 96: 122-133.

3 Hamilton JG, Lobel M, Moyer A: Emotional distress following genetic testing for hereditary breast and ovarian cancer: a meta-analytic review. Health Psychol 2009; 28: 510-518.

4 Pieterse AH, van Dulmen S, van Dijk S, Bensing JM, Ausems MG: Risk communication in completed series of breast cancer genetic counseling visits. Genet Med 2006; 8: 688-696.

5 Bradbury AR, Dignam JJ, Ibe CN et al: How often do BRCA mutation carriers tell their young children of the family's risk for cancer? A study of parental disclosure of BRCA mutations to minors and young adults. J Clin Oncol 2007; 25: 3705-3711.

6 Gaff CL, Clarke AJ, Atkinson P et al: Process and outcome in communication of genetic information within families: a systematic review. Eur J Hum Genet 2007; 15: 999-1011.

7 Van Oostrom I, Meijers-Heijboer H, Duivenvoorden $\mathrm{HJ}$ et al: A prospective study of the impact of genetic susceptibility testing for BRCA1/2 or HNPCC on family relationships. Psychooncology 2007; 16: 320-328.

8 Meiser B: Psychological impact of genetic testing for cancer susceptibility: an update of the literature. Psychooncology 2005; 14: 1060-1074.

9 Sivell S, Elwyn G, Gaff CL et al: How risk is perceived, constructed and interpreted by clients in clinical genetics, and the effects on decision making: systematic review. $J$ Genet Couns 2008; 17: 30-63.

10 Michie S, McDonald V, Marteau TM: Genetic counselling: information given, recall and satisfaction. Patient Educ Couns 1997; 32: 101-106. 
11 Watson M, Duvivier V, Wade Walsh M et al: Family history of breast cancer: what do women understand and recal about their genetic risk? J Med Genet 1998; 35: 731-738.

12 Bober SL, Hoke LA, Duda RB, Tung NM: Recommendation recall and satisfaction after attending breast/ovarian cancer risk counselling. J Genet Counsel 2007; 16: 755-762.

13 DiCastro M, Frydman M, Friedman I et al: Genetic counseling in hereditary breast/ ovarian cancer in Israel: psychosocial impact and retention of genetic information. Am J Genet 2002; 111: 147-151.

14 Vos J, Oosterwijk JC, Gomez-Garcia E et al: Perceiving cancer-risks and hereditylikelihood in genetic-counseling: how counselees recall and interpret BRCA 1/2-test results. Clin Genet 2011; 79: 207-218.
15 Somer M, Mustonen H, Norio R: Evaluation of genetic counselling: recall of information, postcounselling reproduction, and attitude of the counsellees. Clin Genet 1988; 34: 352-365.

16 Kwiatkowski F, Girard M, Hacene K, Berlie J: Sem: a suitable statistical software adaptated for research in oncology. Bull Cancer 2000; 87: 715-721.

(c) This work is licensed under the Creative Commons SOMERIHIS RESERVED Attribution-NonCommercial-No Derivative Works
3.0 Unported Licence. To view a copy of this licence, visit http:// creativecommons.org/licenses/by-nc-nd/3.0/ 